\title{
A Novel Experience in Diagnosing Bone Lesions by FNAC
}

\author{
Dr. K. Rekha MD ${ }^{1}$, Dr K R Umadevi M D ${ }^{2}$ \\ ${ }^{\mathbf{1}}$ Associate Professor, Department of Pathology, Madha Medical College \&RI ,Kovur Ch- \\ ${ }^{2}$ Professor \& Head of Department of Pathology, Madha Medical College \& RI,Kovur ,Ch-
}

\begin{abstract}
We evaluated the diagnostic accuracy of fine-needle aspiration biopsy in a retrospective study of 80 cases over the past three years-April 2013 to March 2016. All cases of primary and suspected local recurrence of a primary bone tumor or a metastatic lesion were included. We analyzed our experience with FNA in bone lesions, thesewere cases presenting in Tertiary health care Hospital and primaryhealth care centers. Fine-needle aspiration biopsy of bone lesions was performed as an outpatient procedure. The cases were grouped into two major classes 1) Benign lesions including infectious conditions 2) Malignant lesions. Cytopathological diagnoses of our cases correlated well (95\%) withHistopathological and radiological features. The present study and article is a shared experience of pathologists from two different geographic zones (W Nigeria and S India) with a variety of bone lesions and clinical presentations. Satisfactory aspirate which was considered conclusive for cytological evaluation was obtained from 72 of the 80 patients. Of the 8 failures, there were 5 aspirates with insufficient yield and 3 in which a diagnosis could not be made. The inconclusive aspirates (3/80) were obtained from other remote clinics and were suboptimal for process and evaluation. One of them was a rare interesting case which was acase foreign body implant, an indigenous bullet injury in a freak accident from a remote tribal area. The radiological picture and clinical details enabled conclusion in this particular case. Among the 72 cases 33 were Malignant and 39 were benign lesions. The malignant lesions correlated well with the radiological and histomorphological studies. (Table 1) Our diagnosis was correct in95\% of the cases.
\end{abstract}

Keywords: FNAC, Cytological correlation, Osteogenic Sarcoma , Benign and Malignant

\section{Introduction}

The term bone tumor is a broad category, encompassing benign and malignant neoplasm, reactive focal abnormalities, metabolic abnormalities, and miscellaneous "tumor like" conditions. The clinical diagnosis of Bone lesions has always been an algorithmic approach based on anatomical location and age of presentation. With the evolution of evidence based practice, correlation with cytomorphological features has gained much significance.

The prevalence of many individual specialty clinics where the initial diagnosis and screening alone is conducted has led to an increased demand for a cytological screening of tumors This cytomorphological evaluation can give an early correlation and confirmation of clinical diagnosis and enable decision for surgical and radio therapeutic management .

The tables represented in this article enable understanding of the different variety of bone lesions commonly referred to pathologist for fine needle aspiration (Table 2). A major portion of these cases are shared from experience at a primary center with only basic management modalities. The purpose was not only to enable confirmation or ruling out of malignancy in these lesions, the procedure proved to be of much help in planning further management and referral to higher centers. A correlation with cytomorphological features of these lesions becomes almost mandatory to recognize the different lesions at various locations in different age groups to enable the best line of management.Knowledge of this information alone is enough to narrow the differential diagnosis considered approximate.

\section{Objective}

Fine needle aspiration cytology (FNAC) has in recent times gained clinical recognition for evaluating skeletal lesions. We have analyzed our experience with FNA in bone lesions with emphasis on areas of difficulty and limitations, from cases presenting in private organizations with limited facilities. The purpose was not only to enable confirmation or ruling out of malignancy in these lesions, the procedure proved to be of much help in planning further management and referral to higher centers.

\section{Materials and Methods}

Over a period of three years FNA was performed in 80 cases of bone lesions. Aspirations were performed in complete aseptic precautions, by cytopathologists using 22-gauge needle after a thorough explanation of the procedure and availing consent. In our study, we used both air-dried smears and $96 \%$ alcohol fixed smears. The air dried smears were stained by Leishman stains and alcohol fixed smears stained by the Hematoxyllin and Eosin method. This enabled a better appreciation of the nuclear morphology

The material obtained was subjected to Leishman, Giemsa, and Hematoxyllin and Eosin staining. The smears were assessed for cellularity under Giemsa. The morphological features were observed under both leishman and hematoxyllin stains. Out of 80 cases unsatisfactory aspirate was obtained in 3 cases. Cytohistological correlation was available in cases. Ref Table 1

Table 1: Benign and Malignant categorization

\begin{tabular}{|c|c|c|}
\hline Total & Benign & Malignant \\
\hline 72 & $54 \%$ & $46 \%$ \\
\hline
\end{tabular}




\section{International Journal of Science and Research (IJSR) \\ ISSN (Online): 2319-7064}

Index Copernicus Value (2013): 6.14 | Impact Factor (2015): 6.391

Table 2: The various lesions with frequency of occurrence

\begin{tabular}{|c|c|c|c|c|}
\hline $\begin{array}{c}\text { S. } \\
\text { No }\end{array}$ & Diagnosis & $\begin{array}{c}\text { Benign/ } \\
\text { Malignant }\end{array}$ & $\begin{array}{c}\text { Frequency of } \\
\text { occurrence in } \%\end{array}$ & $\begin{array}{c}\text { Gender } \\
\text { M:F }\end{array}$ \\
\hline 1 & Haemangioma & Benign & 20 & $1: 1$ \\
\hline 2 & Chondroma & Benign & 23 & $1: 2$ \\
\hline 3 & Giant cell tumor & Benign & 24 & $2: 1$ \\
\hline 4 & Ganglioneuroma & Benign & 10 & $1: 1$ \\
\hline 5 & Foreign body & Rare condition & 3 & - \\
\hline 6 & Cystic Lesions & Non tumor lesion & 20.5 & $2: 1$ \\
\hline 7 & Osteosarcoma & Malignant & 82 & $2: 1$ \\
\hline 8 & Multiple myeloma & Malignant & 18 & $2: 1$ \\
\hline
\end{tabular}

\section{Result}

Among the 72 cases analyzed 39 cases were benign (inclusive of non-tumor and cystic lesions ) and 33 cases were malignant. The malignant cases were Osteosarcoma 27. Multiple myeloma 6. Of the 39 Benign lesions, Haemangiomas were 8, Chondroma 9, Giant cell tumors 10, Ganglioneuroma 4,Cystic lesions 8 and a rare foreign body 1 , were categorized. $95 \%$ of these cytological diagnosis correlated well with radiographic and histomorphological evaluation. The distribution and frequency of occurrence in the cases we analyzed is represented in Table 2. Interestingly our study of the FNAC bone lesions did not have any infectious conditions. The majority benign lesions were of giant cell tumor and among malignant variety Osteogenic sarcoma were predominant

\section{Discussion}

Fine needle aspiration is adopted as a routine procedure of evaluation in bone lesions both primary and secondary metastatic lesions (4). It has emerged as a cost effective tool for initial diagnosis. The distinct cytological features enable confirmation of a suspected malignant lesion by its radiological and anatomical presentation. However with the evidence based practice it has become mandatory for a cytological evaluation and thus FNA correlation is much sought.

The retrospective analysis of the eighty cases over a period of three years has shown a good correlation of the FNAC interpretation with that of the final diagnosis. This correlation of FNA in bone lesions is of much value to plan for both pre-operative and post-operative management in bone tumors.

Also the awareness of a preliminary evaluation by FNA for a definite plan of action in management of the condition helps the surgeon in preparing the patient well ahead of the elective procedure to be adopted. A well explained FNA procedure educates the patient and family members enabling a better acceptance of the procedure and management upto rehabilitation.

Fine needle aspiration technique has become a successful diagnostic tool, in the diagnosis of bone tumors. With adequate yield of aspirate, it is possible to detect the classical morphological features of the various types of bone lesions.

The cytological features of FNA in most bone tumors have been reported previously Many bone tumors have palpable soft tissue extensions, hence the choice of the length of the needle to do FNA depends on the size of the lesion along with the plain radiograph. In most cases the site of aspirate resulted in yield from vascular and reactive zones causes challenge in reporting.

In our study, we used both air-dried smears and $96 \%$ alcohol fixed smears. The air dried smears were stained by May Grunwald Giemsa stains and alcohol fixed smears stained by the Hematoxyllin and Eosin method. This enabled a better appreciation of the nuclear morphology.

Giant cell tumour another common benign tumour of bone had the typical cytological picture of dispersed cells and cohesive clusters with mononuclear single oval to spindle cells and large multinucleated osteoclast type giant cells along with spindle cell clustering. In many of our cases the yield showed variable osteoclastic clusters in a hemorrhagic background. Fig 1 The nuclei of the multinucleate giant cells were uniform and bland. The correlation of cytopathological features along with clinical presentation and plain radiograph were helpful to categorize the lesions.

In our study Osteosarcoma had the highest incidence in malignant tumors'. The clinical features of age at presentation, progressive growth of lesion, X-ray findings with an osseous defect, cortical destruction, sun burst appearance were all taken into correlation. The microscopic features of Osteogenic sarcoma were confirmed with the cytological features of hyperchromatic cells with pleomorphic nuclei, prominent nucleoli, along with large multinucleated Fig 2.tumour giant cells. The detection of the osteoid matrix admixed with loose or cohesive clusters, mitotic figures was of great interest.

The aspirates with microscopic features of increased cellularity, abnormal plasma cells, ranging from isolated to binucleate forms, pleomorphic cells with abundant eosinophilic cytoplasm and a characteristic perinuclearhoff were suggestive of myeloma .The radiological and clinical presentation were also correlated. Fig 3.

FNAC of the lesion showingpleomorphic cells with predominance of binucleate forms and perinuclear halo in a background of fibrillarychondroid material were diagnostic of Chondrosarcoma. Fig4.

The difficulties encountered in many of our cases were, the blind approach to the clinically presenting mass, usually with a hemorrhagic aspirate, achieving the correct anatomical localization of the tumors and the reliance on morphological criteria was a good challenge. However these were overcome with the correlation of cytopathological features with imaging studies and clinical details.

\section{Conclusion}

We conclude from our study of 80 FNA cases of bone lesions over a three year period that FNA can be employed with good clinical and plain radiological input to arrive at a preliminary diagnosis in patients with bone tumors'. FNAC proves the most useful tool in the pre-operative assessment of bone tumours. It is of much help in planning the ideal 


\section{International Journal of Science and Research (IJSR) \\ ISSN (Online): 2319-7064}

Index Copernicus Value (2013): 6.14 | Impact Factor (2015): 6.391

surgical procedure in the individual cases that may require post surgical support like prosthesis or implants.

\section{Acknowledgements}

MsChinnasa , MrRexan ,MrSubash ,Dr Ankush G, Dr Jha, Dr NileshDr RP Singh, Radiology Department Primus Superspeciality International Hospital,Abuja.

\section{References}

[1] Text book of Cytopathology, 1st Edition by Grace T. McKee MRCPath (Author)

[2] Rosai and Ackerman's Surgical Pathology - 10th Edition by Juan Rosai(Author)

[3] Fine needle aspiration cytology of bone tumours--the experience from the National Orthopaedic and Lagos University Teaching Hospitals, Lagos, Nigeria Obiageli E Nnodu, SO Giwa, Samuel U Eyesan, Fatima B Abdulkareem.

[4] Kabukcuoglu F, Kabukcuoglu Y, Kuzgun U, Evren I: Fine Needle Aspiration of malignant bone lesions.ActaCytol 1998, 42: 875-882.

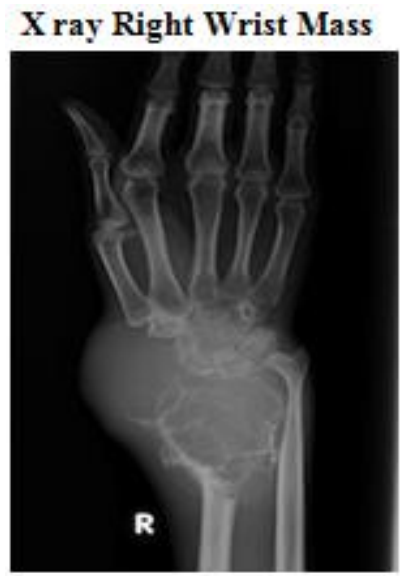

$\mathrm{X}$ ray Left Humerus Mass

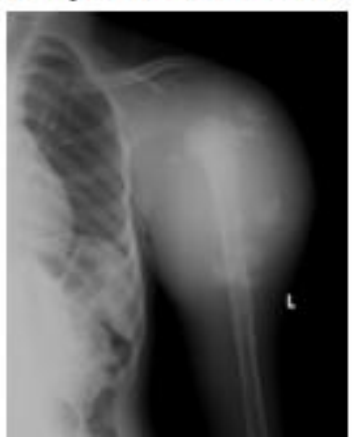

Sternal Mass $60 / \mathrm{M}$

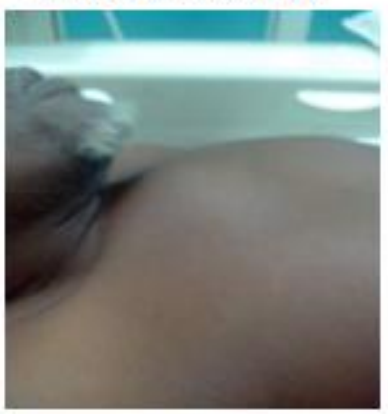

Figure 1

Right Wrist Mass

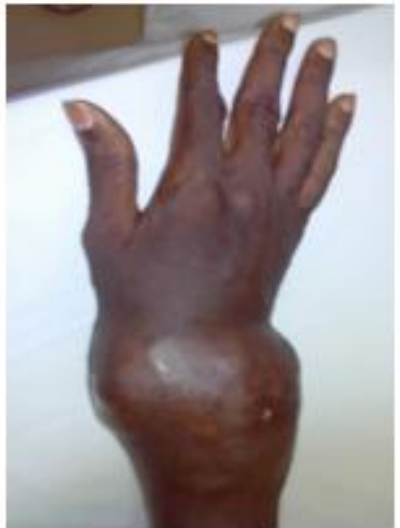

Figure 2
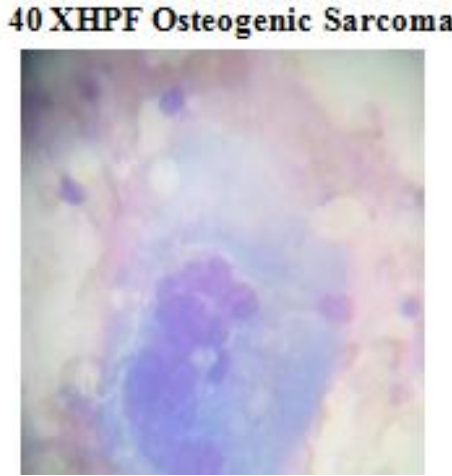

Figure 3
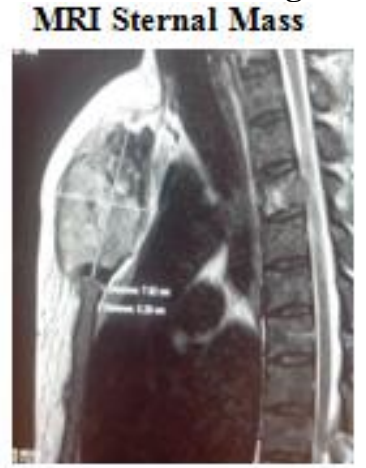

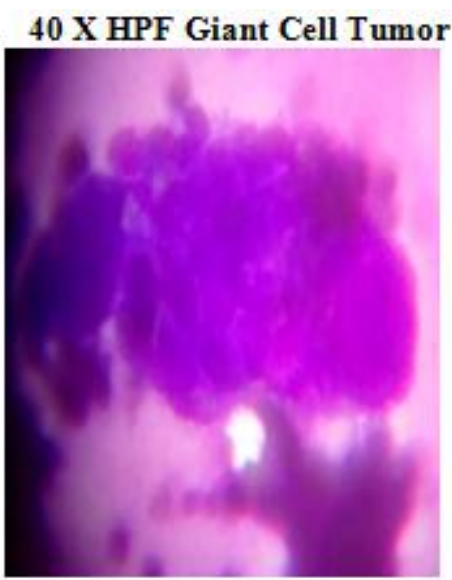

$40 \mathrm{X}$ HPF Osteogenic Sarcoma

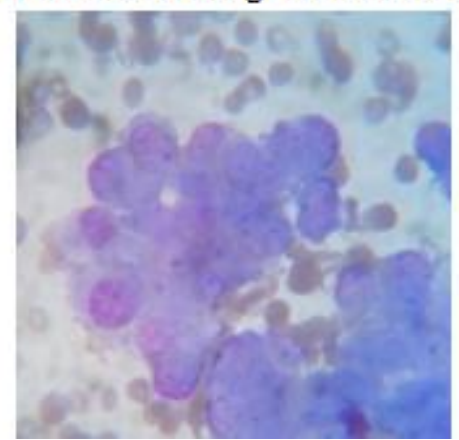

10 X \& 40X HPF Multiple Mỵeloma

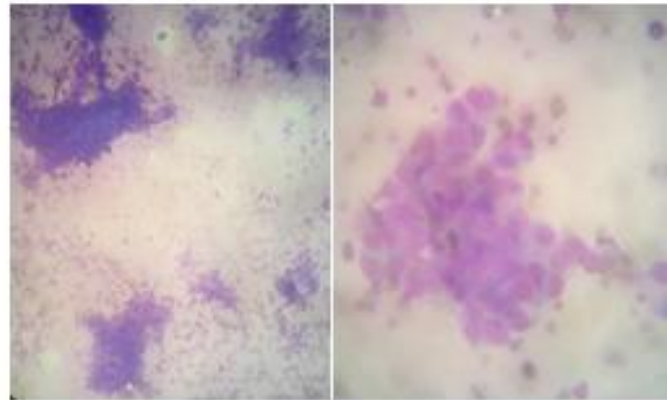


International Journal of Science and Research (IJSR)

ISSN (Online): 2319-7064

Index Copernicus Value (2013): 6.14 | Impact Factor (2015): 6.391

Figure 4

$\mathrm{X}$ ray Right Femur Mass

10X HPF Chondrosarcoma

40X HPF Chondrosarcoma
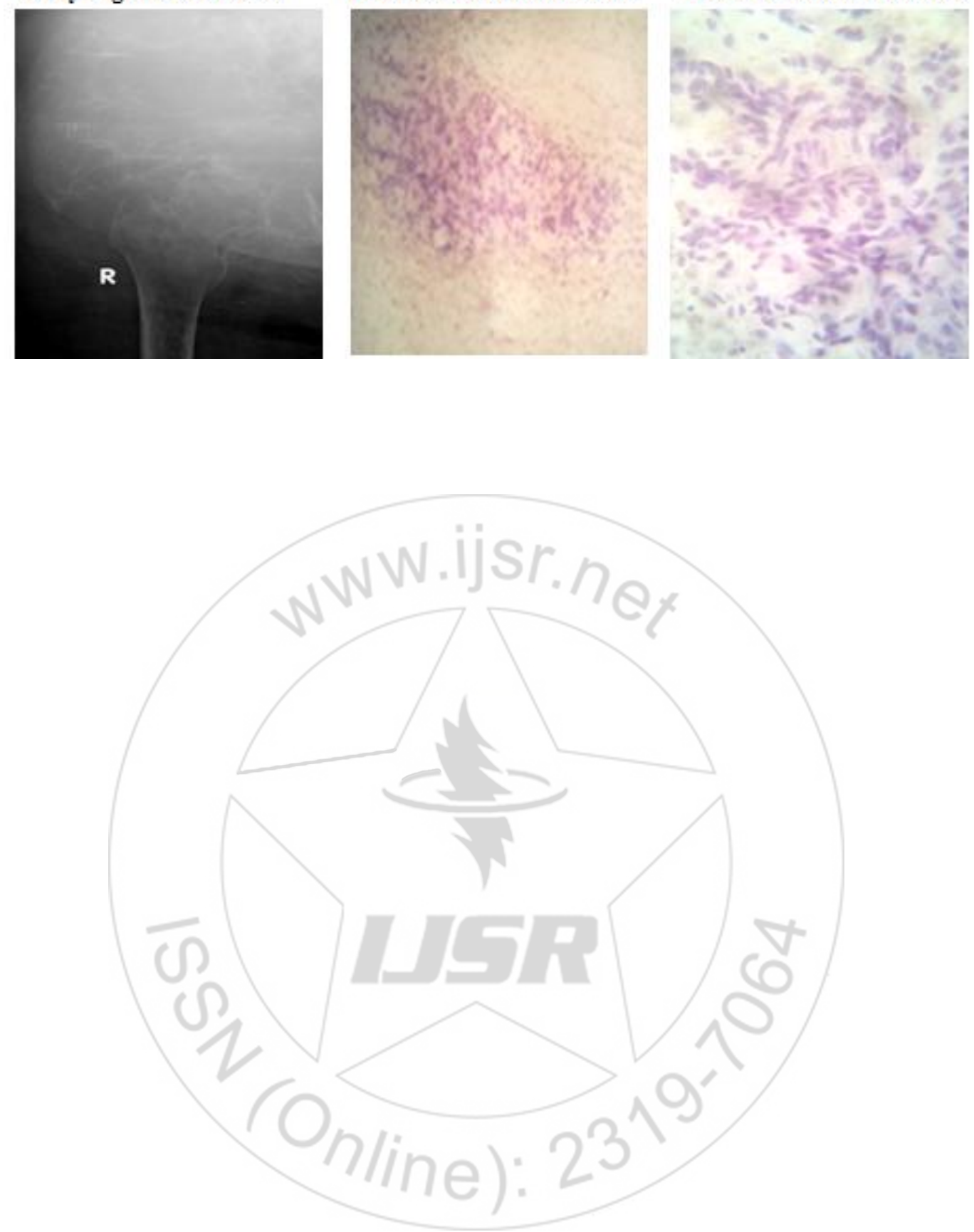

Volume 5 Issue 5, May 2016

www.ijsr.net 